\title{
A Craft Approach to Health Awareness in Children
}

\author{
Swamy Ananthanarayan ${ }^{1}$, Katie Siek², Michael Eisenberg ${ }^{1}$ \\ ${ }^{1}$ Department of Computer Science \\ University of Colorado Boulder \\ Boulder, CO, USA \\ \{ananthas, michael.eisenberg\}@ colorado.edu \\ ${ }^{2}$ School of Informatics and Computing \\ Indiana University \\ Bloomington, IN, USA \\ ksiek@indiana.edu
}

\begin{abstract}
Children in the United States are increasingly at risk for a vast array of health problems due to poor dietary habits and lack of physical activity. In recent years, a burgeoning landscape of wearable and mobile health technologies in the form of activity trackers and fitness applications have focused on addressing this problem. While these solutions have had some measure of success with children, there is also evidence that youngsters are not readily adopting the types of fitness implements that adults find useful. In this paper, we present a computational toolkit that blends craft and health, permitting children to craft their own tangible health visualizations based on data from an accompanying wearable device. We also present the results of an encouraging 2-month study conducted with middle school children. The results suggest that craft could potentially serve as a gateway to healthful thinking in children.
\end{abstract}

\section{ACM Classification Keywords}

H.5.m. Information Interfaces and Presentation (e.g. HCI): Miscellaneous

\section{Author Keywords}

Children's health; craft technology; wearable computing; health visualization

\section{INTRODUCTION}

The need for instilling health awareness in children is more essential than ever considering the prevalence of childhood obesity in the United States. In the past three decades, obesity has tripled among children and adolescents [24]. This trend is concerning because it opens the door for life threatening chronic conditions such as diabetes and high blood pressure [12]. Apart from the physical health issues they face, a recent report by the CDC suggests that mental disorders among children are also on the rise [29]. Thus, helping children towards "healthful thinking" is crucial to minimizing health risks in the future.

Permission to make digital or hard copies of all or part of this work for personal or classroom use is granted without fee provided that copies are not made or distributed for profit or commercial advantage and that copies bear this notice and the full citation on the first page. Copyrights for components of this work owned by others than ACM must be honored. Abstracting with credit is permitted. To copy otherwise, or republish, to post on servers or to redistribute to lists, requires prior specific permission and/or a fee. Request permissions from permissions@acm.org.

DIS 2016, June 4-8, 2016, Brisbane, Australia.

Copyright (C) 2016 ACM ISBN 978-1-4503-4031-1/16/06 ...\$15.00.

http://dx.doi.org/10.1145/2901790.2901888
While we are fundamentally faced with a health issue, it is a problem that is tightly interwoven with present culture. As American historian Steven Mintz highlights, there has been a gradual shift over the past century in children's culture from outdoors to bedrooms, and from improvised toys to fantasy toys marketed directly to children [22]. These shifts have led to an increase in sedentary, electronically mediated play and a decline in unstructured play [22]. Therefore, a solution to the health problem must perturb and counteract prevalent culture.

Since children are increasingly comfortable with technologies such as mobile phones, portable music players, and tablet computers, researchers have started exploring the role of technology in encouraging healthy behaviors. In recent years, general health and wellness technologies have made their way to children. Activity trackers coupled with online gaming and rewards platforms (e.g., Sqord, iBitz, Striiv) are available on the consumer market for kids. Increasingly, there are also motion gaming consoles such as Microsoft Kinect, Nintendo Wii, Sony PlayStation Move that utilize motions based on player movements to interact with characters on-screen. Many researchers have used these commercial activity trackers and motion consoles to create exertion interfaces $[3,11]$ and to test social theories of behavior change with children [21, 35].

These commercial health technologies and research platforms are enjoying some measure of success with children, especially when designed with social and peer support in mind [21]. However, there is also evidence to suggest that kids are not naturally taking to the types of fitness implements that adults commonly use. In a recent Northwestern University study on teens' use of technology for health, they found that only $7 \%$ of teens used wearable health devices and only $1 \%$ reported any behavior change from their use [37].

It seems that what is needed is a different way of thinking about health technologies for children. In prior work, we proposed a framework of ambient and wearable computing where users can craft their own personal meaningful visualizations of health [1]. While the framework we described did not target a particular user group, we feel the health crafting idea is particularly salient to children because it gives them a sense of control over their environment. Moreover, crafting is already a part of children's culture. In this paper, we focus on our efforts to substantially extend the health crafting framework for children. We present a computational toolkit of ambient and wearable building blocks where children can (a) track a particular health or wellness metric through a wear- 
able device, and (b) relay that information to a set of ambient computationally enhanced blocks. These blocks abstract the information and present them to the child using a variety of feedback modalities such as light, sound, or movement. Thus, children can create different health visualizations by combining and crafting these blocks in unique highly personalized ways. In addition and perhaps most important, we present the results of an extensive 2-month evaluation of the system with a group of middle school children.

\section{RELATED WORK}

There are several strands of research that have strongly influenced the design and motivation of our work. In this section, we examine a subset of prior work that includes existing health technologies for children, tangible health visualizations, the constructionist approach, and advances in craft technology. In many ways, it is only because of progress in these areas that our work is possible.

\section{Games for Health}

In recent years, health and wellness technologies have seen meteoric growth in both academia and industry. Wearable activity trackers like the Fitbit and mobile phone health applications (e.g. MyFitnessPal) are increasingly common in today's culture. While much of this work has focused on motivating and supporting healthy behaviors in adults, new technological health interventions are emerging to engage children. In this section, we concentrate primarily on general health and wellness technologies since it is closer in line to our own work.

Much like the adult versions, wearable activity trackers have started to appear in child friendly packages on the consumer market. While they are not considerably different from those for adults, they do offer a gaming element that is age appropriate. Sqord (sqord.com), for example, couples a wrist worn activity tracker with an online application where kids can create their own avatar to earn medals and points in a socially networked system. Similarly, Ayogo (ayogo.com) connects the bioluminescence of a virtual jellyfish to an individual's heart rate. In a skillful combination of heart beats per minute and mobile phone tilts, the player steers her way through an aquatic world with obstacles and challenges. While a majority of health interventions for children present themselves as games, a few such as Zamzee (zamzee.com) and Geopalz (geopalz.com) utilize a wearable tracker with an online rewards platform. Children, in this case, use points earned from physical activity to buy various prizes and items at online stores.

The gaming element is also common in academic research. Snack Educator [16] for example, aims to improve the snacking habits of low socioeconomic children through a set of playful and culturally sensitive mobile phone games. In the realm of physical activity promotion, Berkovsky et al.'s Play Mate! system [3], leverages a Nintendo Wii console to sense players' movements and move an on-screen marble through a 3D world. Although these types of exertion interfaces [23] were originally developed for adults, they could potentially be used to motivate children. Perhaps the most common device appropriated for promoting physical activity in children has been the pedometer. Persuasive Audio, for example, uses a simple pedometer to control and vary music tempo based on activity level [9]. StepStream [21] and Chick Clique [35] on the other hand, leverage community and social interaction among children to motivate higher step counts.

While many of these technologies have had some success in motivating healthy habits, we offer an alternative path that might be better suited to children's culture. Personal crafted health technologies could become meaningful possessions that could enrich a child's sense of narrative history. Technology in this case, is capable of having sentimental or autobiographical significance. It is in this aspect that the blending of craft and health might prove to be a valuable contribution.

\section{Tangible Visualizations}

The majority of existing approaches to health and wellness technologies target the virtual medium. Although, these screen based interfaces support dynamic data updates and favorable data visualization constructs, they focus primarily on the visual and auditory system. As Ullmer and Ishi point out, these virtual interfaces often neglect the other senses [36].

While physical objects have long been used for information presentation, they have only recently been adopted for health data. Two recent examples include SweatAtoms [17], and Activity Sculptures [34]. In both cases, physical activity data was transformed into 3D printed artifacts. The researchers found that material artifacts made participants more conscious of their physical activity involvement. Prior work has also examined the use of tangible metaphors to motivate healthy outcomes. BreakAway [14] employed the body language of a small desktop sculpture to make people aware of their sedentary lifestyle. In a similar vein, MoodWings [19], utilized a small wearable butterfly to make users aware of their stress levels. Specifically for children, Parés' work on the Interactive Slide [33] and the Interactive Water Installation [28] highlights tangible playground designs that couple physical activity with social interaction.

The ambient visualizations and Parés' playground designs are compelling examples because they blend sensory stimuli, such as sights, sounds, textures, aromas and motion, aspects that are hard to replicate on a mobile phone or computer screen. Moreover, the concreteness of artifacts offers various social affordances that virtual objects typically don't. Among children for example, tangible objects can be traded, or put on a shelf for display.

\section{Constructionist Approach}

More broadly, our work is clearly undertaken in the tradition of "constructionism" pioneered by Seymour Papert. Learners in this view are seen as constructors of their own knowledge. Thus, materials and concepts are supposed to help children to construct personally meaningful "objects to think with" that bridge the gap between the sensory and abstract worlds [26]. An important element of Papert's philosophy includes the public nature (the presentation and sharing) of artifacts that children create. Resnick et al. build on these ideas and argues that construction kits should help children explore important 
concepts and domains through their expressive activities [32]. The learning objective then is not to "teach" health explicitly, but rather to empower children towards real world activities. Papert describes this notion of empowerment as the opposite of "learning by being taught" [27]. Thus, the aim of our work is to offer children new health learning experiences with novel tangible technologies. This paradigm focuses less on adult ideas and more on children's voices.

\section{Craft Technology}

Perhaps the most influential precursor to our work has been in the area of craft technology - that is, the idea of interweaving computation with common craft materials such as paper and textiles. This blending often takes many diverse forms and yields craft objects with some form of embedded intelligence. Traditionally, craft has been separate from the context of electronics, but recent growth in open source electronic platforms such as Arduino (arduino.cc), LilyPad [4] and Adafruit's FLORA (adafruit.com/flora) have lowered the barrier to entry and facilitated the confluence between these two disparate fields. Already, within the span of a few years, a variety of child-friendly construction kits are available, including peeland-stick electronic circuits for interactive craft [10], sewable electronic modules for wearable systems [15], and magnetic modular electronics for young inventors [2].

In addition to these construction kits, researchers have explored new methods for embedding traditional electronics in common craft materials such as paper, wood, and textiles [5, 20]. These methods employ a variety of conductive ingredients (e.g. inks, paints, glues, tapes, fabrics, yarns) to create electrical circuits in non-standard mediums. Notable examples of these hybrid craft practices include, an interactive electronic pop-up book [30], electronics enriched storytelling with conductive ink [13], and paper animation with shape memory alloys [31].

Our work follows this rich research tradition, and uses craft technology as a medium for expressing health. We employ and build on these methods to showcase how children can develop low-cost electronic visualizations of their health.

\section{SYSTEM OVERVIEW}

An early version of the framework and a sample implementation is described in [1]. In this paper, we provide a brief overview along with significant updates to the system. Specifically, we have manufactured custom PCBs for the wearable base using surface mount components and developed three more ambient feedback modules. The wearable and ambient modules are presented as a health crafting toolkit for children.

Before delving into the details, we provide a simple scenario of how a child might use the system to track outdoor exposure and reflect that data in an origami windmill. The child begins by crafting the wearable device with the UV sensor into an accessory that she can carry, attach or wear. Then she crafts the origami windmill utilizing the ambient motor module. Next, she wears or carries the wearable with her and spends a certain amount of time outside. Then as she returns home and approaches the origami windmill the cumulative time spent outside is communicated wirelessly from the wearable to the

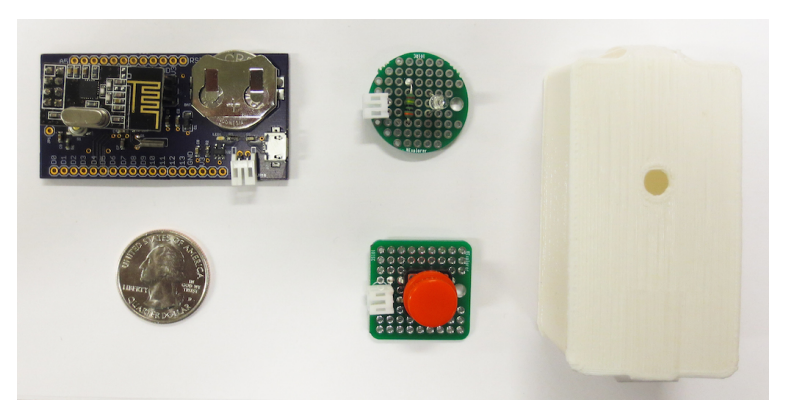

Figure 1. Wearable base with coin cell battery and RF wireless module pictured on the left, UV sensor (top) and button (bottom) pictured in the middle, 3D printed case to house the device pictured on the right.

motor module. In this example, the motor module and thus the origami windmill spins faster as more time is spent outside.

\section{Wearable Monitor}

For gathering health and wellness data, we developed a custom wearable health monitor that is comprised of three modules: a microcontroller base, a UV sensor module for tracking time spent outside, and a button for manually tracking various userdefined health habits (Figure 1). The base is a custom printed circuit board manufactured and built with surface mount components. It is designed to be single sided so it can lay flat against various surfaces. Integrated in the board is a microcontroller, a coin cell battery holder, a wireless radio transceiver and an optional lithium ion charging circuit. The wearable base keeps track of time by using a watch crystal and the microcontroller's real-time counter. When connected to a sensor module, it periodically time stamps sensor events and stores the data in the microcontroller's internal memory. In part, due to the limited number of components and aggressive power saving modes, the device is small, low power and can run on a single coin cell battery for weeks. In order to accommodate other health sensors, the sensor module is separate from the microcontroller base. In our particular example, we showcase two external modules (UV sensor and button), but envision our design as a starting point towards a larger, more varied health crafting ecosystem.

The entire wearable device is craftable, and the child can choose to make it a part of their daily wear as they deem fit. For children less inclined to craft, we provide a simple 3D printed case (Figure 1) that can be used as a housing for the wearable. We designed our own custom board because existing off-theshelf solutions (such as the Lilypad) were not developed with power consumption in mind. We needed a self-contained wearable sensor platform that could potentially be used in a long-term study. Although the board is custom, we utilize an Arduino bootloader to enable users to write programs using the Arduino IDE. By using the Arduino software we make use, not only of professional-grade software and documentation, but also the vibrant and growing community of Arduino users.

\section{Ambient Modules}

The ambient visualization portion of this system is comprised of a master microcontroller (Arduino UNO) coupled with a wireless transceiver that receives aggregated sensor data 


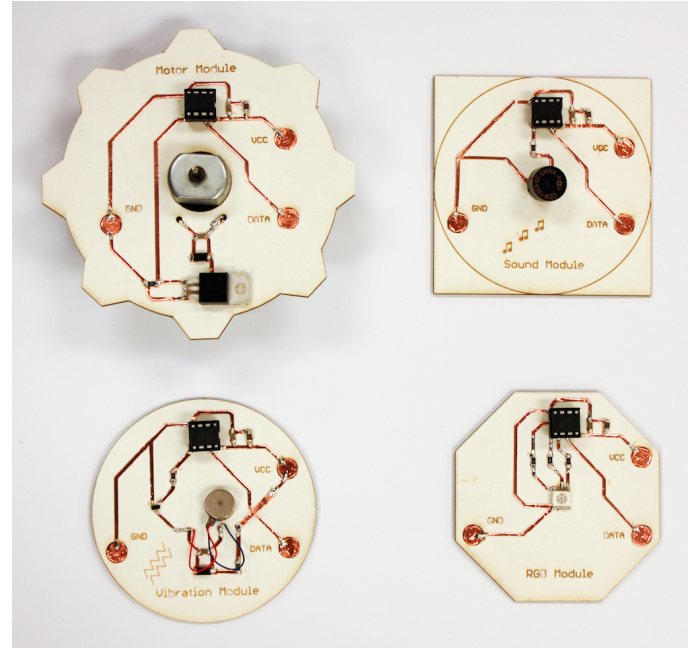

Figure 2. Ambient paper based modules for visualizing health data. Clockwise from top left: DC motor module, Sound module, RGB LED module, Vibration Module

from the wearable tracker. Based on the data, the master controller issues commands to a set of feedback nodes on a shared serial communication network. These feedback nodes in turn actuate devices such as DC motors, buzzers, speakers, LEDs or vibration motors to help visualize the health data.

A key aspect of our design lies in the object oriented concept of polymorphism, the ability (in programming) to represent the same interface for different underlying forms. In our case, the concept is implemented in paper circuit blocks where each block is controlled by a tiny microcontroller that is preprogrammed with a specific node ID and pre-defined behaviors for that particular block. Figure 2 shows the four node types that are part of the system. The nodes actuate a DC motor, a piezo speaker, a RGB LED, and a vibration motor. Each node is constructed from card stock, copper tape and a few electronic components. The shape of each module suggests its use (e.g. motor module is shaped like a gear) and the copper traces are cut using an inexpensive desktop craft cutter. We implemented these nodes in paper (as opposed to custom PCBs) since paper is an extremely versatile, cost-effective, craft-friendly, and expressive medium. Moreover, it is childfriendly and can tolerate mishaps that tend to occur while children are playing or learning.

In order to function, each node typically requires just three connections: power, ground, and data. Once power and ground are connected, the node automatically displays its default behavior. For example, the motor module slowly increases its rotational speed; the RGB module cycles through all the colors; the sound module plays the built-in animal sounds; and the vibration module slowly oscillates faster. We employ magnetic connectors to facilitate easy solder-less connections to the nodes. The copper encased neodymium magnets not only help hold the module against a surface, but also provide sound electrical connections. Thus, nodes can be combined or used individually to create a health visualization.
From a software perspective, each node is responsible for actuating the feedback modality it controls based on data received over the serial bus. In the case of the RGB LED node, it transitions from red to green to indicate a positive health behavior. It accomplishes this task by mapping the red to green color gradient to the wearable sensor value range. In a similar fashion, the motor node maps its rotational speed to the sensor value range. Thus, across different node types we get consistent, appropriate behavior for the health data received from the wearable device.

A notable aspect of our design is the division of labor between the wearable device that tracks health data and the ambient modules that help visualize that data. This separation increases the breadth of possible visualizations by opening up the full craft domain ${ }^{1}$. Additionally, it also allows us to offload some of the power limitations faced by wearable devices in general. Much like the wearable, the ambient modules also use the Arduino platform for programming.

\section{STUDY OVERVIEW}

Since this was our first field trial with children, we were fairly open-ended in our approach. Our intent was to see whether and how children would use our system, rather than conduct a classical assessment of intervention versus non-intervention. We were particularly interested in their perceptions of health, any difficulties they faced during the construction process, the types of visualizations they created for representing health, and the subsequent use of the system. Lastly, we also wanted to explore if there were any social aspects to the crafting process with children potentially co-crafting a single ambient visualization.

\section{Study Location}

Our study was conducted in collaboration with a local afterschool enrichment program that provides interest based education in arts and technology for children aged 10-18. The children in this community are typically from low income families. About 60 percent of them participate in free and reduced lunch programs. In order to better understand the cultural and social practices within the clubhouse, we volunteered over 150 hours (over the span of a year) tutoring and mentoring children prior to conducting the study. Volunteering also helped in building trust and rapport with the youth and the clubhouse staff members.

Children typically attend the clubhouse on a drop-in basis Monday through Friday from 3 p.m. to 7 p.m. While the clubhouse serves a wide age range of children, the demographic is skewed towards middle school children who mix freely with those younger and older than themselves. The clubhouse has minimal structure and students individually identify projects that are important to them, and set out learning how to use various art and technology skills to complete the project. The adult instructors and mentors help facilitate student interests. The clubhouse follows a democratic education process where learning is self-initiated and self-motivated. We observed a

\footnotetext{
${ }^{1} \mathrm{~A}$ video of four illustrative health visualizations may be seen at: https://vimeo . com/102302357
} 
strong maker culture that revolves around the practice of creating fine and graphic visual art pieces. This practice is slowly starting to incorporate 3D sculptures with the recent addition of a 3D printer.

\section{Methods}

During our volunteering sessions over the year, we conducted both formal and informal workshops on paper circuits, soldering, and Arduino programming based on clubhouse members' requests. In many ways, these sessions laid the foundation for the study by introducing children to basic electronics and programming.

We acquainted children with our work three months prior to the study during clubhouse meetings based on advice from staff members. During these meetings, we showcased the modules, the wearable, a few sample visualizations, and answered children's questions. This not only helped generate interest, but also identified potential participants. With the help of clubhouse staff, we then recruited 11 participants between the ages of 11 and 14 with a regular attendance record. Of these 11, 9 participants, consisting of 6 boys and 3 girls, completed the full study; one participant moved to a different part of the state, and the other withdrew due to family issues. Participants already had some exposure to modern technologies having used personal computers for digital art projects at the clubhouse. Moreover, a majority of them reported owning a computer at home and using it for videos, music, and photos. All of the children had prior experience with basic electronics and crafting, either through courses at the clubhouse or personal projects.

After recruitment, we conducted two 45-minute design sessions with the participants to facilitate ideation and to better understand how they might use our system. During these group discussions, children explored health related issues such as what it means to be healthy, activities they considered important and metrics they would like to monitor. Participants also investigated potential visualizations for their health activities. A communal poster served as a focal point for the discussion and the sketches.

The design sessions were followed by a month long prototyping and development phase where children crafted and programmed their wearables and associated visualizations. We initially alloted only 2 weeks for this process but found that children usually attended the clubhouse on alternating days of the week (Monday and Wednesday or Tuesday and Thursday) and required more time to craft their systems. When receiving the wearable device, we asked that children take time to craft the device into an artifact of their choosing (e.g. backpack, headband), considering their daily lifestyles and their own fashion and artistic sensibilities. We suggested that they choose an artifact they used everyday so that they would not forget the device at home. Additionally, they were instructed on the operation of the device, the location of the sensor or the button and how to activate it. During this time period, participants also crafted tangible visualizations using the various ambient modules. Children decided what modules they wanted to use, how it would visualize data from the wearable (e.g. should the motor spin faster or slower?), and what story it would tell. Participants were given all the craft materials they needed (felt, fabric glue, Velcro, cardstock, etc.) to complete these tasks. Moreover, researchers and clubhouse staff were always on hand to assist children with crafting, programming, and debugging.

Following the development phase, children evaluated the systems they created for 2 weeks. Participants took the crafted wearable home with them, while their visualizations remained at the clubhouse. The main reason for this decision was to minimize any technical issues children might face and to ensure proper functioning of the system. To avoid collisions between different wearables and visualizations, we programmed unique identifiers into each child's wearable and ambient module(s). This way, the visualizations only responded to the associated wearable.

The entire study lasted approximately 2 months and was approved by the Institutional Review Board at our university. We conducted contextual and semi-structured interviews, maintained field notes, and video recorded children's experiences during the development and evaluation phases. At the end of the study, participants were given a $\$ 15$ dollar retail gift card for their time and effort.

\section{RESULTS}

Overall, the health crafting approach was positively received by the clubhouse, with participants creating an electronic health mural using the ambient modules. Children were generally less interested in adult notions of health and tracked self-selected behaviors they considered to be healthy. Volunteering and building rapport with the clubhouse community was definitely helpful in gathering candid feedback from the children. In this section, we discuss the ambient visualization that children created (a community health mural), the wearables they crafted, and their ideas of health during the process.

\section{Notions of Health}

Central to our work is the idea of health and what it means to be healthy. While as adults, we subscribe to the common mantras of diet and exercise, it was clear from the beginning of our study that children had somewhat different notions. In our initial group discussions with children, participants often cited diet and physical activity as the two most important attributes of good health. However, when we asked kids why they really thought so, they often mentioned their parents or health education classes at school as the source. Although they acknowledged that they needed to eat more fruits and vegetables, and exercise frequently, they were less interested in these traditional behavioral indicators. A 12-year-old girl (P9) commented, "I know exercise is good for you. My mom is always telling me to go play outside, but sometimes my friends aren't there. What am I supposed to do? Run around? That is no fun." In general, participants had very little concern over their health and described themselves as fairly healthy. As one 13-year-old boy (P3) candidly summarized, "Listen [researcher's name], honestly [pause] I am not all that interested in health. I like this project because I like playing with electronics and gadgets." In our study, this participant 

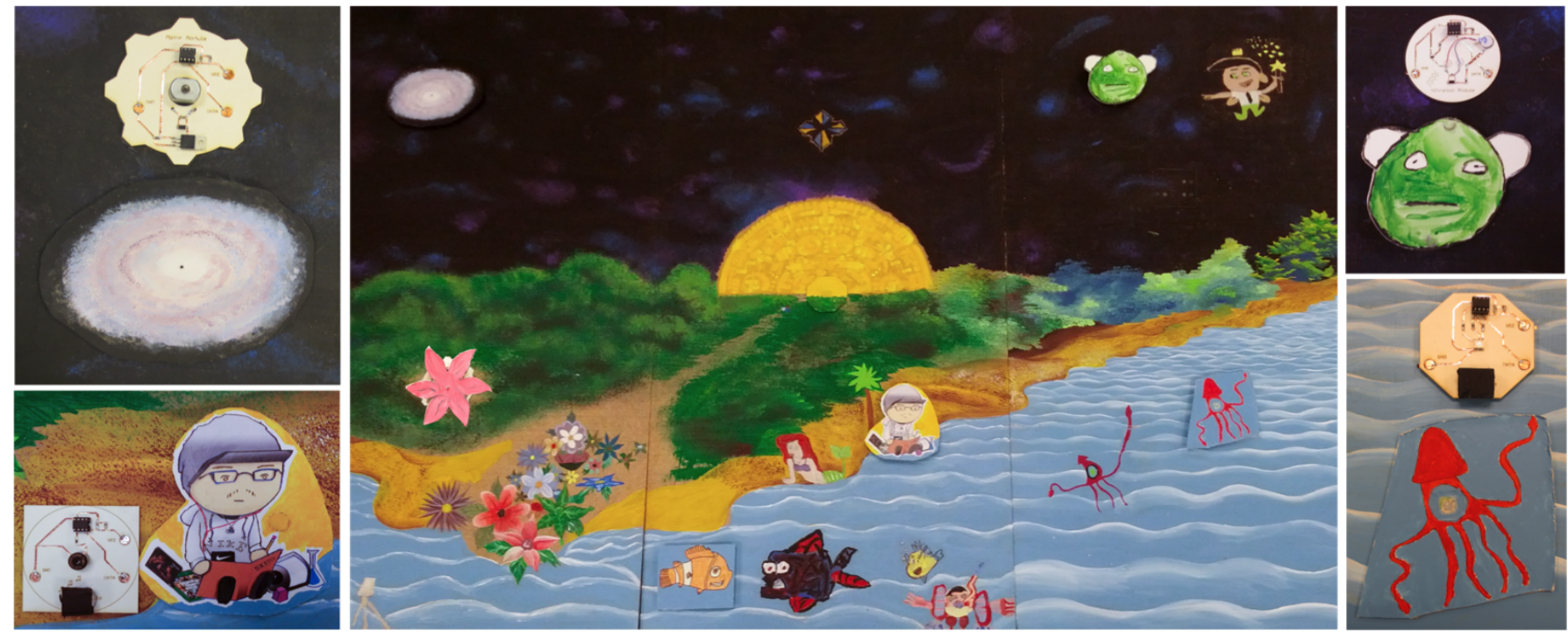

Figure 3. Electronic health mural created by middle school children using ambient modules. Each module reacts to the respective owner's wearable device. Examples of each of the four types of modules are highlighted on the left and right sides of the mural. On the left, the galaxy spins using the motor module and the boy seated on the beach plays a tune using the sound module. On the right, the mask vibrates and the octopus changes color.

\begin{tabular}{l|l|l|l|l}
\hline P\# & Sex & Age & Health Metric & Mural Artifact \\
\hline P1 & M & 12 & Fruit consumption & Pinwheel (Motor) \\
\hline P2 & M & 11 & Water consumption & Tiki Mask (Vibration) \\
\hline P3 & M & 13 & Reading & Octopus (LED) \\
\hline P4 & M & 12 & Music, guitar & Nemo (LED) \\
\hline P5 & M & 13 & Playing outside & Cosmo (LED) \\
\hline P6 & M & 14 & Sketching & Self-portrait (Sound) \\
\hline P7 & F & 11 & Playing outside & Galaxy (Motor) \\
\hline P8 & F & 12 & Coloring, drawing & Ariel (Sound) \\
\hline P9 & F & 12 & Music, making tunes & Sun (LED) \\
\hline
\end{tabular}

Table 1. Participant information and health metric they chose to track and the mural artifact they created

chose to track his reading habits instead. He expressed, "I been trying to read more. I like it because it makes me feel good afterwards."

Perhaps the single central idea that children commonly voiced about health was the ability to participate in activities which they considered important. In their view, being able to perform these activities served as markers of health. Thus, the majority of participants chose activities related to their well-being. Table 1 summarizes the health/wellness metric each participant chose to track. Only two participants in this table (P1 and P2) chose traditional dietary measures. Even in the case of P2, his desire for tracking the number of times he drank water everyday was somewhat misinformed. When we asked him why he wanted to track water consumption, he explained, "I heard in school that our bodies are mostly made of water. So putting water in must be good." The 12-year-old male participant (P1) however, clearly identified a health case for eating fruits. He said, "Well eating fruits is better than eating Cheez-Its. This way you don't get fat."

Regardless of the metric children chose, they welcomed the idea of crafting their health. When asked to discuss why they preferred this approach as opposed to being given an off-theshelf pedometer or activity tracker, they cited the expressive nature of the project. As one participant (P2) commented, "You put the stuff that you like on it...it expresses how I feel. Instead of other people doing all the work, it has the stuff that I like...that I put on."

\section{Health Mural}

It was clear from the initial design sessions that the participants were part of a tightly knit social group. Thus, it did not come as much of a surprise when children suggested building a clubhouse mural that represented the community at large. They proposed an electronic mural where each participant could contribute a crafted ambient module that adhered to a common larger theme. The modules in this vision would only react to the respective owner's wearable device.

During the initial design sessions, participants brainstormed various common themes for the mural where they attempted to complete the sentence, "Our clubhouse is like a...". The answers included, human body, solar system, ocean, rain forest, gang of super heroes, Christmas tree, and jungle. In subsequent meetings, these ideas coalesced into a core set of themes that included an ocean, a night sky, and a jungle. Since participants could not agree on a single theme, they decided to create a scene (Figure 3) that included aspects of the core themes.

The mural in Figure 3 is approximately 6' $\mathrm{x} 4^{\prime}(1.83 \mathrm{~m} \mathrm{x}$ $1.22 \mathrm{~m})$ and is composed of 3 pieces of tempered hardboard. It houses various ambient modules that magnetically connect to the surface of the acrylic painting. Small holes in the hardboard connect the modules to the copper tape based circuitry housed in the back. Thus, participants were able to craft their visualizations independently of the mural and magnetically connect them as they finished. Children decided where to place their modules and clubhouse staff members facilitated the connections to the circuitry in the back.

The images on the left and right side of Figure 3 showcase individual visualizations created using each type of module. 


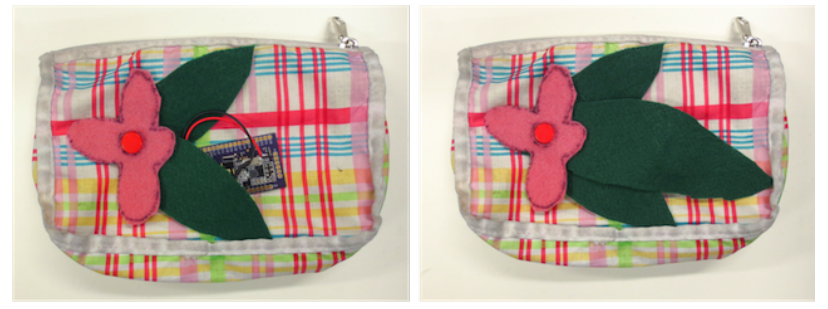

Figure 4. Pencil pouch with a felt flower design that houses the button and microcontroller base. The button is hidden in the eye of the flower.

For the sake of illustration, the crafted card stock pieces are placed next to the module rather than on top as shown in the mural. The galaxy on the upper left utilizes the motor module to spin at a different rate based on data from the UV sensor. The young boy seated on the beach (a self-portrait) employs the sound module to play increasing bars of the Mario Brothers theme song as its owner creates more drawings in his sketchbook (tracked with the button). The visualization on the top right, is a 11-year-old boy's version of a vibrating Tiki mask; it responds to the number of cups of water he drinks in a given day. Lastly, the eye of the octopus changes color from red to green as its owner spends more time reading. Table 1 highlights the mural artifacts created by each of the participants.

The mural was very well received by the children, with participants describing it as "awesome" and "cool." Two of the participants were keen on showing it to their parents as they came to pick them up. Although progress on the mural was initially slow, it gradually intensified over time since contributions were publicly visible. It should be noted that even though we only recruited 9 participants, the mural was a clubhouse effort. Children younger and older contributed to various aspects of the mural, such as painting the background, even though they were not involved in the study.

We definitely observed a great deal of social interaction around the mural, especially as it was starting to take shape. Participants would often be motivated by each others work. P4 and P5, who were close friends, would often see the progress of the other and work on their own ambient modules. P4 would often ask, "What did [P5] work on last time? His thing [Cosmo cartoon character] is getting better every time I see it." Children also learned from each other on the best ways to accomplish various goals. For example, with the RGB LED module, we taught a few children how to diffuse light using vellum paper (a type of translucent paper). These children were often helpful in disseminating this knowledge to others working on similar LED modules. Even when children were finished with their respective modules, they would contribute to other aspects of the mural, such as painting bushes, flowers or adding stars. As one 11-year-old girl (P7) summarized, "It is nice to make something for the clubhouse together. We spend so much time here."

\section{Wearables}

The idea of crafting wearable devices to track health received a somewhat mixed response. Individual designs in this space generally fell into two camps: those that focused exclusively

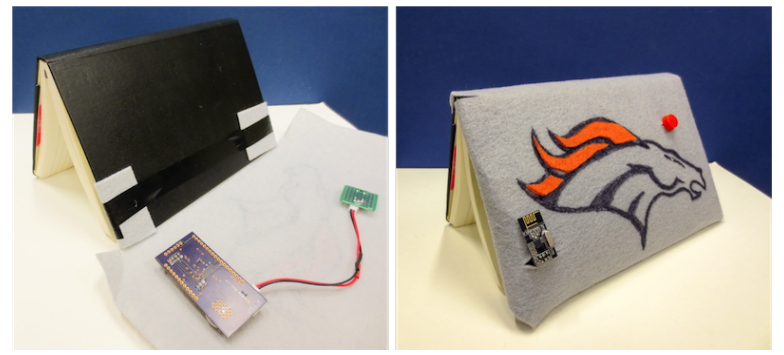

Figure 5. A sports themed book cover for a sketch book that houses the button and microcontroller base. The button and wireless module extend through the felt.

on functionality; and those driven by appearance and aesthetics. Generally, the students who had favorable prior experiences with electronics (5 participants) from robotics clubs or otherwise focused on functionality, while crafters and artists (4 participants) identified with aesthetics.

The participants who associated with appearance and aesthetics were hesitant to start crafting the wearable, citing their minimal experience with electronics and programming. However, once it was explained to them that the wearable was already programmed and that it only required their choice of sensor, they were more willing. Participants who were uncomfortable with electronics definitely needed some assurance that staff members and researchers would be there to help them if they were stuck. This group produced some of the most beautiful designs of the study. Figure 4, showcases a pencil pouch with a felt flower designed by a 12-year-old girl (P8) who was interested in tracking her coloring habits. The button that is used to track the habit is artfully hidden in the pistil or eye of the flower. Since the pencil pouch holds her coloring pencils, the flower accessory is aptly placed as a reminder. Similarly, another burgeoning artist (14-year-old boy, P6), designed a felt book cover for his sketchbook (Figure 5) to track his daily sketching practice. As an avid sports fan, the book cover highlights his favorite sports team mascot. A somewhat more playful design by a 11-year-old girl (P7) is shown in Figure 6. This panda backpack accessory, created with felt, fabric glue, and safety pins utilizes the UV sensor to track her time spent playing outside. Rather than permanently fastening the wearable device to her backpack, the participant wanted something she could easily transfer from bag to bag.

Participants who were primarily interested in the functionality of the device did not spend as much time crafting the wearable. They wrapped their devices inconspicuously using whatever

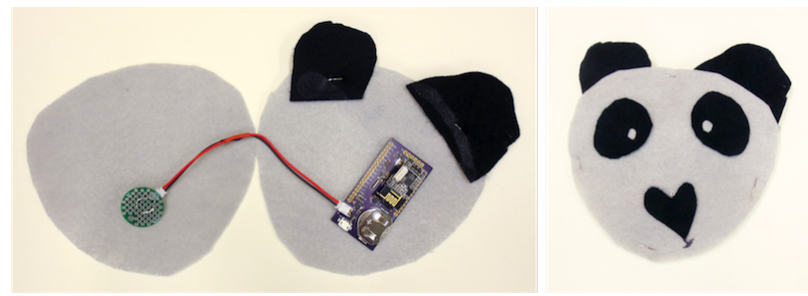

Figure 6. A panda bear backpack accessory that houses the UV sensor and the microcontroller base. 
materials were furnished or used the provided case. Although the wearable did not require much programming, other than selecting the necessary sensor in the Arduino programming environment, functionality-driven participants were more willing to experiment and see how it worked with their crafted visualizations.

Perhaps, the most interesting aspect of the wearable device was that it was hardly used (strictly speaking) as a "wearable." Children primarily used it as an accessory to items they already had or carried it like their mobile phones. When we probed into this issue, children cited the difficulty of making something truly wearable. As one participant (P7) commented, "Well if I want to add it to my shoe, I gotta think about all sorts of things...if I add to my backpack, it is just a lot easier." Another participant brought up the issue of context and articulated, "Why would I want to make it wearable? I want to track how much I read."

Overall, while the wearables worked as expected, we did face a number of issues while deploying them. Children often did not securely fasten the wearables. As a result, we had to replace two of the wearable boards due to accidents. Another issue we experienced was with the button module; there was a tendency for a lot of false positives since the button could be easily triggered. The pencil pouch and the book examples faced this issue since they were usually carried with other items in a backpack. We had to create a small plastic cover to protect the button in these cases.

\section{Crafting and Programming}

Children were very comfortable with crafting the wearable and the ambient modules. As one 12-year-old girl (P8) said, "Well I have painted, glued stuff, and sewed and done all these things before at the clubhouse, so it wasn't that hard." There was however, a tendency to focus on the ambient modules more than the wearable. We attributed this outcome to the social and public aspects associated with creating the mural as opposed to the individual nature of crafting the wearable. This idea was endorsed by P6, the oldest participant, who commented, "Well I spent a lot more time drawing the self portrait on the computer because it was something others would see and I wanted it to look good. The [sports team] cover on my sketching book, that was just for me."

Although children were expressive and creative with the craft portion surrounding the technology, they were less inclined to modify or program the technology itself. With the ambient modules, children often accepted the default behaviors changing only minor aspects of the system such as the color transition scheme of the RGB LED module or the spin rate of the motor in the Arduino programming environment. A few participants however, did spend considerable time customizing their ambient modules. For example, P6 and P8 wanted the sound modules to play specific theme songs. P6 wanted the Mario theme song played for the self-portrait he had created and P8 wanted the Little Mermaid theme song played for Ariel the mermaid. While the participants found the theme songs online and converted it to usable tones for use with the ambient microcontroller (with help from clubhouse staff), they needed considerable help programming the ambient module. They found the programming aspect frustrating and confusing at times, but these two participants were heavily motivated to get their songs playing. The resulting modules however, received many accolades from their peers. P8 remarked, "Oh my god, my friends thought this was so cool! I can't believe I made that!"

With respect to the wearable, participants often chose the default behavior associated with the UV sensor or the button. Even though the wearable base could store sensor data for multiple weeks, and the ambient modules could respond to aggregated weekly data, participants chose only to reflect the current day's data. Thus, the ambient module would only respond to the button presses or the outdoor exposure data for the prevailing day. In a sense, children expected the wearable and ambient modules to simply work. Although children in our study were generally less interested in programming the system, they were appreciative of being able to track what was important to them. As one 12-year-old boy (P4) commented, "I've been trying to get better at playing the guitar for a while now. Sometimes I forget, sometimes I have too much homework. It is nice that I can use this [referring to the wearable and the Nemo ambient module] to remind me."

\section{System Usage}

During the 2-week trial, we observed a general excitement around the mural as children approached and examined their ambient artifacts. Participants were well aware of their connection to the mural and often commented on how the artifact changed in response to their behavior. P8 remarked, "I drew and colored today... and I noticed that after I pressed the button, the Little Mermaid theme song played more." Children had a tendency to use the wearable more the day they were attending the clubhouse. For some participants this was because they were more likely to perform their self-selected activities during clubhouse hours. Activities such as drawing/sketching (P6, P8), painting/coloring (P8), or recording music (P9) were generally clubhouse related activities. For other participants, the clubhouse and the mural served as a reminder to work on their self-selected wellness habits. As P1 articulated, "I ate 3 fruits today. I knew this thing [referring to the pinwheel] wouldn't spin otherwise."

We also observed that participants who invested more time crafting the wearable, generally used it to a greater extent when compared to their less devoted counterparts; this was true of all 4 participants who spent more than 90 minutes (approximately two clubhouse visits) crafting their wearable. Usage was defined with respect to each participant's goals. So for example, if a child used the button to track a personal goal of 3 fruit servings per day, and she pressed the button 3 times on any given day, we would label that as sufficient or good use of the system ${ }^{2}$.

We identified two reasons why usage was higher with this group: 1) The device was contextually crafted and was convenient to use; and 2) Participants had a clear sentimental or

\footnotetext{
${ }^{2}$ We acknowledge that this an imperfect method of measurement given that we do not have an established baseline for each participant. However, it is useful for just getting a general relative idea of usage.
} 
personal attachment to the crafted object. For both P6 and P8, the button for tracking their respective sketching and coloring habits was located on the object required for their practice; in the case of P8 the button was conveniently located on the color pencil bag (Figure 4) and for P6 the button was on the sketching book itself (Figure 5). As P6 confirmed, "It is hard not to press the button...it is right there [referring to the sketching book]." The other two participants, P7 and P4, expressed a more personal attachment to their crafted devices. The 11-yearold girl (P7), who crafted the panda bear backpack accessory (Figure 6) expressed, "It is so cute. I love carrying it around". The 12-year-old boy (P4), recalled a personal family memory from when he was younger.

We saw light to moderate use of the system with participants who were less interested in crafting the wearable. We noticed that personal goals were occasionally met, especially on clubhouse days. Participants who used the button to track selfselected behaviors, tended to use the device in batch mode, often pressing the button multiple times at the end of the day. Initially, we were concerned that children were perhaps gaming the system, or had some misplaced sense of obligation to us. However, when we questioned them as to their usage patterns, they often cited misplacing the device at home or forgetting to press the button. As one of our most straightforward participants, P3, remarked, "I did read my book 3 times today. I just forgot to take it [the wearable] with me in the morning. So I pressed the button 3 times at night before bed."

When we asked participants to reflect on their health crafting experiences, they expressed joy at being able to see a personalized reaction from the mural. From a wellness perspective, one participant likened the mural to a reminder system. P9 commented, "Well it is nice to see something change because you did something. Sometimes you forget and it helps you remember. I know my galaxy didn't spin for a couple of days, cause I didn't do nothing [laughs]" Another 13-year-old boy (P5) mentioned the tangible and public nature of the mural, "I mean it is huge and it is right there. Everyone can see if your piece ain't shinin, that you didn't do anything." Lastly, during the two week evaluation, we observed a slight decline in system use as children became acclimated to the mural. However, by the end of the study, many of the participants were already considering making modifications or adding other ambient modules.

\section{DISCUSSION}

Our study provided promising results in support of the health crafting approach for middle school children. Although the craft portion of the study was more popular among children, we did find healthful thinking among participants. Several key themes emerged from our experiences and observations.

\section{Is it Really Health?}

In our study, this notion of health and what it means to be healthy was questioned and interpreted differently by children. Traditional adult measures such as diet and physical activity often took a backseat to more personal wellness metrics. Children chose, coloring habits, time spent playing outside, or playing musical instruments as representative measures of health. While one may argue that this is not health, we strongly feel that it is important to start thinking about health from a child's perspective. Moreover, it could be argued that the wellness metrics that children chose are an important aspect of mental health. Reading because you "feel good afterwards" (P3) or wanting to play the guitar more (P4) are holistic measures that account for individual and personal habits. Even as adults, we have habits that might not be related to physical health but are crucial to our well being. Talking to our grandparents for example, or building airplane models might very well be the activities that give different individuals a sense of calmness in their daily lives. Moreover, we highlight the World Health Organization's (WHO) definition of health established over half a century ago. It states, "Health is a state of complete physical, mental and social well-being and not merely the absence of disease or infirmity [25]." The metrics that children chose certainly fall within this definition of health.

For children, these wellness metrics may be a great starting point for thinking about more traditional health metrics in the future. Rather than forcing a particular health agenda on children, we strongly feel that having them think about their own health, in a manner they are comfortable with, is a necessary stepping stone towards the types of health activities (e.g., running, eating more fruits and vegetables) we as adults consider necessary. Strategies such as goal setting learned by children during this process are transferable to other activities in the future. A child that learns how to set reasonable goals for improving their sketching habits, may use that same skill in learning how to eat more vegetables in the future. Even in our modest study, there was some evidence of children reflecting on their goal setting processes. The 13-year-old boy who wanted to improve his reading habits reflected on how he should have set his daily reading goal to twice rather than thrice a day. We argue that this awareness and understanding of one's own thought processes is important for establishing any kind of health behavior in the future.

\section{Wearable Device Design: Context and Automation}

As alluded to earlier, an interesting result of our study was that the wearable device was scarcely used as a wearable. To some extent, this was a consequence of the types of activities children wanted to monitor. Monitoring your fruit consumption habits, as one 12-year-old boy wanted (P1), hardly requires a wearable tracker. However, what struck us as fascinating was how important context was to the successful use of the device. With two of our children, the wearable was crafted directly to an object related to the activity they wanted to track. The pencil pouch with the felt flower design for tracking coloring habits and the sports themed book cover for tracking sketching habits were both wonderful examples of context sensitive design. Both these participants were excellent users of the system despite having to enter all their data manually.

One of the issues with manual data entry is that users often forget to enter their activity or write down their progress. To address these issues, technologists and researchers have raced towards automating data collection. However, another approach might be to attach electronic devices to artifacts that are contextually related to the activity. Instead of having a 
mobile phone and entering data periodically, a simple device attached to an contextually related object might serve as a good reminder to enter data for activities that are typically hard to automatically track.

Additionally, the manual entry of data might help users ultimately reflect on the activity they are doing. We must acknowledge, however, that there is a tradeoff here; if we do not automate data collection enough, then we place a heavier burden on the user to manually track the information. But if we automate too much, then we rob the user of opportunities for reflection. It may be that children who did not contextually craft the device may have benefited from automated data collection. However, other researchers in the area of personal informatics have found that a full automated system sometimes hinders users from keeping track of and making sense of their physical activity data [18]. The researchers suggested areas for further study including the need to explore an appropriate balance of automated technology and user control. In the case of children, a manual approach might be helpful in guiding them in the habits of reflection.

\section{Supporting Creativity in Health Maintenance}

First, the health crafting style, unlike most efforts in wearable health electronics, emphasizes the creative input of the user. The children's projects shown here are not exemplars of "convenient" or "invisible" health maintenance, nor are they intended to be. Instead, they are intended to tap into an impulse for creative play that supports a high level of individual decision making. In this constructionist vision, the child appropriates the possibilities of craft technologies for the purposes of understanding and maintaining her own physical well-being.

There is something of a distinct emotional tone in our work relative to that suggested by many commercial wearable devices. Commercial devices are often advertised as a "product for everyone" where the underlying image is one of "control" or "mastery." A certain urgency is implied in the statements: "Every moment matters", "Because fitness is the sum of your life", "How far will you go?", and "Live healthier and be more productive $e^{3 "}$. Contrast this with the health crafting approach which emphasizes informality, play, and a sense of whimsy about health maintenance; the task of maintaining one's body is thus considerably more expressive and considerably less demanding. We witnessed this informal, playful attitude in the language children used during our evaluations. Words such as "cute," "cool," and "awesome" were common when discussing the mural or the individual trackers.

Essentially, we view health crafting as a form of creative play that has the potential to introduce serious health concepts. Moreover, since the child has taken the initiative to design his own challenging visual representations of health or wellness, it is at least plausible that the health crafting approach will evoke meaningful participation and "buy-in" for health maintenance. Our field trial were promising in this aspect considering that we were working with early adolescent children, a challenging group for any research study.

\footnotetext{
${ }^{3}$ Quotes from fitbit.com, jawbone.com/up, microsoft.com/ microsoft-band
}

\section{“Pleasantly Frustrating” Experiences}

In many ways, our work in health crafting is at a very early stage. Even though the wearable and ambient modules have undergone multiple revisions, in our view, the wearable can still be made smaller, the connectors to the sensors can be more robust, and the programming environment can be made friendlier (to name a few). Needless to say, running studies with early-stage, non-production systems can be challenging, especially with children. However, we discovered that the hassles children faced, as a consequence of our less-robust system, often lead to satisfying experiences. The difficulty in figuring out Arduino programming, or finding the right power and ground points, exposed the inner workings of the system, and children in turn struggled and learned. The satisfaction of struggling and making the artifact was ultimately the reward for some of the participants. P8, who used the sound module to play the Little Mermaid theme song, found the programming aspect confusing and frustrating. However, when she finished, she exclaimed, "I can't believe I made that."

This is not to say our system is perfect the way it is. It can certainly be improved, but there is a trade-off here between making things too easy, too obvious and exposing all the details. As Gross and Eisenberg argue, there is a indeed a "fine balance between eliminating needless complexity to make a more elegant design environment, and hiding important detail in the name of ease-of-use [8]." In essence, the technological environment must encourage curiosity and mastery, and mastery almost always implies a struggle or a challenge. As Csikszentmihalyi points out "a challenging activity that requires skill" is one of the components of an "optimal experience [6]." However, if the experience is too challenging then children may get frustrated. On the other hand, if it is too easy, children may lose interest and value. What is needed is a kind of "pleasant frustration" [7], where challenges feel hard but doable. This balance is indeed hard to strike.

\section{CONCLUSION}

In this paper, we presented a framework of wearable and ambient modules that enables children to create their own personally meaningful representations of health. To evaluate our design, we conducted a 2-month study with 9 middle school children between the ages of 11 and 14 . The children designed and implemented an electronic health mural that represented the community at large while displaying an individual's health. Participants' ideas of health revolved around being able to perform activities which they considered important. Thus, the majority of participants tracked activities related to their well-being. Overall, children were expressive with the craft surrounding the technology, even though they used the technology in a rudimentary fashion. The results suggest that crafting could be a possible gateway to health.

\section{ACKNOWLEDGMENTS}

This work is supported by the National Science Foundation Award No IIS-1231645. We wish to thank the clubhouse staff for their continued support and help. 


\section{REFERENCES}

1. Swamy Ananthanarayan, Nathan Lapinski, Katie Siek, and Michael Eisenberg. 2014. Towards the Crafting of Personal Health Technologies. In Proceedings of the 2014 Conference on Designing Interactive Systems (DIS '14). ACM, New York, NY, USA, 587-596. DOI : http://dx.doi.org/10.1145/2598510.2598581

2. Ayah Bdeir and Ted Ullrich. 2011. Electronics As Material: LittleBits. In Proceedings of the Fifth International Conference on Tangible, Embedded, and Embodied Interaction (TEI '11). ACM, New York, NY, USA, 341-344. DOI : http://dx.doi.org/10.1145/1935701.1935781

3. Shlomo Berkovsky, Mac Coombe, Jill Freyne, Dipak Bhandari, and Nilufar Baghaei. 2010. Physical Activity Motivating Games: Virtual Rewards for Real Activity. In Proceedings of the SIGCHI Conference on Human Factors in Computing Systems (CHI '10). ACM, New York, NY, USA, 243-252. DOI : http://dx.doi.org/10.1145/1753326.1753362

4. Leah Buechley and Michael Eisenberg. 2008. The LilyPad Arduino: Toward Wearable Engineering for Everyone. IEEE Pervasive Computing 7, 2 (April 2008), 12-15. DOI : http://dx.doi .org/10.1109/MPRV. 2008. 38

5. Leah Buechley and Hannah Perner-Wilson. 2012. Crafting Technology: Reimagining the Processes, Materials, and Cultures of Electronics. ACM Trans. Comput.-Hum. Interact. 19, 3, Article 21 (Oct. 2012), 21 pages. DOI : http://dx.doi.org/10.1145/2362364.2362369

6. Mihaly Csikszentmihalyi. 1991. Flow: The Psychology of Optimal Experience. Harper Perennial, New York, NY.

7. James Paul Gee. 2005. Learning by Design: Good Video Games as Learning Machines. E-Learning and Digital Media 2, 1 (2005), 5-16. DOI : http://dx.doi.org/10.2304/elea.2005.2.1.5

8. Mark D. Gross and Michael Eisenberg. 2007. Why Toys Shouldn't Work "Like Magic": Children's Technology and the Values of Construction and Control. Digital Game and Intelligent Toy Enhanced Learning, IEEE Intl. Workshop on 0 (2007), 25-32. DOI :

http://dx.doi.org/10.1109/DIGITEL . 2007.55

9. Jeffrey Hartnett, Pearl Lin, Lillian Ortiz, and Lindsay Tabas. 2006. A responsive and persuasive audio device to stimulate exercise and fitness in children. In $\mathrm{CHI}$ '06 extended abstracts on Human factors in computing systems - CHI EA '06. ACM Press, New York, New York, USA, 1837. DOI :

http://dx.doi.org/10.1145/1125451.1125799

10. Steve Hodges, Nicolas Villar, Nicholas Chen, Tushar Chugh, Jie Qi, Diana Nowacka, and Yoshihiro Kawahara. 2014. Circuit Stickers: Peel-and-stick Construction of Interactive Electronic Prototypes. In Proceedings of the 32Nd Annual ACM Conference on Human Factors in Computing Systems (CHI '14). ACM, New York, NY, USA, 1743-1746. DOI :

http://dx.doi.org/10.1145/2556288.2557150
11. Johanna Hoysniemi. 2006. International Survey on the Dance Dance Revolution Game. Comput. Entertain. 4, 2, Article 8 (April 2006). DOI :

http://dx.doi.org/10.1145/1129006.1129019

12. Elina Hyppönen, Suvi M Virtanen, Mike G Kenward, Mikael Knip, and Hans K Akerblom. 2000. Obesity, increased linear growth, and risk of type 1 diabetes in children. Diabetes care 23, 12 (December 2000), 1755-1760.

http://view.ncbi.nlm.nih.gov/pubmed/11128347

13. Sam Jacoby and Leah Buechley. 2013. Drawing the Electric: Storytelling with Conductive Ink. In Proceedings of the 12th International Conference on Interaction Design and Children (IDC'13). ACM, New York, NY, USA, 265-268. DOI : http://dx.doi.org/10.1145/2485760.2485790

14. Nassim Jafarinaimi, Jodi Forlizzi, Amy Hurst, and John Zimmerman. 2005. Breakaway: An Ambient Display Designed to Change Human Behavior. In $\mathrm{CHI}$ '05 Extended Abstracts (CHI EA '05). ACM, New York, NY, USA, 1945-1948. DOI :

http://dx.doi.org/10.1145/1056808.1057063

15. Eva-Sophie Katterfeldt, Nadine Dittert, and Heidi Schelhowe. 2009. EduWear: Smart Textiles As Ways of Relating Computing Technology to Everyday Life. In Proceedings of the 8th International Conference on Interaction Design and Children (IDC '09). ACM, New York, NY, USA, 9-17. DOI : http://dx.doi.org/10.1145/1551788.1551791

16. Danish Khan, Swamy Ananthanarayan, Amy Le, Christopher Schaefbauer, and Katie Siek. 2012. Designing mobile snack application for low socioeconomic status families. In Pervasive Computing Technologies for Healthcare (PervasiveHealth), 2012 6th International Conference on. 57-64. DOI : http: //dx.doi.org/10.4108/icst.pervasivehealth.2012.248692

17. Rohit Ashok Khot, Larissa Hjorth, and Florian 'Floyd' Mueller. 2014. Understanding Physical Activity Through 3D Printed Material Artifacts. In Proceedings of the SIGCHI Conference on Human Factors in Computing Systems (CHI '14). ACM, New York, NY, USA, 3835-3844. DOI :

http://dx.doi .org/10.1145/2556288.2557144

18. I. Li, A. Dey, and J. Forlizzi. 2009. Using Contextual Information to Improve Awareness of Physical Activity. In Engaging Data Forum 2009. Boston, MA.

19. Diana MacLean, Asta Roseway, and Mary Czerwinski. 2013. MoodWings: A Wearable Biofeedback Device for Real-time Stress Intervention. In Proceedings of the 6th International Conference on PErvasive Technologies Related to Assistive Environments (PETRA '13). ACM, New York, NY, USA, Article 66, 8 pages. DOI : http://dx.doi.org/10.1145/2504335.2504406 
20. David A. Mellis, Sam Jacoby, Leah Buechley, Hannah Perner-Wilson, and Jie Qi. 2013. Microcontrollers As Material: Crafting Circuits with Paper, Conductive Ink, Electronic Components, and an "Untoolkit". In Proceedings of the 7th International Conference on Tangible, Embedded and Embodied Interaction (TEI '13). ACM, New York, NY, USA, 83-90. D0I : http://dx.doi.org/10.1145/2460625.2460638

21. Andrew D. Miller and Elizabeth D. Mynatt. 2014. StepStream: A School-based Pervasive Social Fitness System for Everyday Adolescent Health. In Proceedings of the 32Nd Annual ACM Conference on Human Factors in Computing Systems (CHI '14). ACM, New York, NY, USA, 2823-2832. DOI : http://dx.doi.org/10.1145/2556288.2557190

22. Steven Mintz. 2004. Huck's Raft: A History of American Childhood. Belknap Press of Harvard University Press,, Cambridge, MA.

23. Florian Mueller, Stefan Agamanolis, and Rosalind Picard. 2003. Exertion Interfaces: Sports over a Distance for Social Bonding and Fun. In Proceedings of the SIGCHI Conference on Human Factors in Computing Systems (CHI '03). ACM, New York, NY, USA, 561-568. DOI : http://dx.doi.org/10.1145/642611.642709

24. Cynthia L Ogden, Margaret D Carroll, Lester R Curtin, Molly M Lamb, and Katherine M Flegal. 2010. Prevalence of High Body Mass Index in US Children and Adolescents, 2007-2008. JAMA 303, 3 (20 January 2010), 242-249. DOI :

http://dx.doi.org/10.1001/jama.2009.2012

25. World Health Organization. 1946. Preamble to the Constitution of the World Health Organization as Adopted by the International Health Conference, New York, 19-22 June, 1946; signed on 22 July 1946 by the representatives of 61 States (Official Records of the World Health Organization, no. 2, p. 100) and entered into force on 7 April 1948. (1946).

26. Seymour Papert. 1980. Mindstorms: Children, Computers, and Powerful Ideas. Basic Books, Inc., New York, NY, USA.

27. Seymour Papert. 1990. A Critique of Technocentrism in Thinking about the School of the Future. Epistemology and Learning Group, MIT Media Laboratory.

28. Narcís Parés, Jaume Durany, and Anna Carreras. 2005. Massive Flux Design for an Interactive Water Installation: Water Games. In Proceedings of the 2005 ACM SIGCHI International Conference on Advances in Computer Entertainment Technology (ACE '05). ACM, New York, NY, USA, 266-269. DOI : http://dx.doi.org/10.1145/1178477.1178523

29. Ruth Perou, Rebecca H Bitsko, Stephen J Blumberg, Patricia Pastor, Reem M Ghandour, Joseph C Gfroerer,
Sarra L Hedden, Alex E Crosby, Susanna N Visser, Laura A Schieve, and others. 2013. Mental health surveillance among children-United States, 2005-2011. MMWR Surveill Summ 62, Suppl 2 (2013), 1-35.

30. Jie Qi and Leah Buechley. 2010. Electronic Popables: Exploring Paper-based Computing Through an Interactive Pop-up Book. In Proceedings of the Fourth International Conference on Tangible, Embedded, and Embodied Interaction (TEI'10). ACM, New York, NY, USA, 121-128. DOI :

http://dx.doi.org/10.1145/1709886.1709909

31. Jie Qi and Leah Buechley. 2012. Animating Paper Using Shape Memory Alloys. In Proceedings of the SIGCHI Conference on Human Factors in Computing Systems (CHI'12). ACM, New York, NY, USA, 749-752. DOI : http://dx.doi.org/10.1145/2207676.2207783

32. Mitchel Resnick and Brian Silverman. 2005. Some Reflections on Designing Construction Kits for Kids. In Proceedings of the 2005 Conference on Interaction Design and Children (IDC '05). ACM, New York, NY, USA, 117-122. DOI : http://dx.doi.org/10.1145/1109540.1109556

33. Joan Soler-Adillon, Jaume Ferrer, and Narcís Parés. 2009. A Novel Approach to Interactive Playgrounds: The Interactive Slide Project. In Proceedings of the 8th International Conference on Interaction Design and Children (IDC '09). ACM, New York, NY, USA, 131-139. DOI :

http://dx.doi.org/10.1145/1551788.1551811

34. Simon Stusak, Aurélien Tabard, Franziska Sauka, Rohit Ashok Khot, and Andreas Butz. 2014. Activity sculptures: Exploring the impact of physical visualizations on running activity. Visualization and Computer Graphics, IEEE Transactions on 20, 12 (2014), 2201-2210. DOI :

http://dx.doi.org/10.1109/TVCG.2014.2352953

35. T. Toscos, A. Faber, K. Connelly, and A.M. Upoma. 2008. Encouraging physical activity in teens: Can technology help reduce barriers to physical activity in adolescent girls?. In Proceedings of the 2nd International Conference on Pervasive Computing Technologies for Healthcare (PervasiveHealth 2008). 218-221. DOI : http://dx.doi.org/10.1109/PCTHEALTH. 2008.4571073

36. B. Ullmer and H. Ishii. 2000. Emerging Frameworks for Tangible User Interfaces. IBM Syst. J. 39, 3-4 (July 2000), 915-931. DOI:http://dx.doi.org/10.1147/sj . 393.0915

37. Ellen Wartella, Vicky Rideout, Heather Zupancic, Leanne Beaudoin-Ryan, and Alexis Lauricella. 2015. Teens, Health, and Technology: A National Survey. Technical Report. Center on Media and Human Development, School of Communication, Northwestern University. 